\title{
Redes y flujos de conocimiento en la acuacultura en el Noroeste de México
}

\author{
Dra. Rosalba Casas Guerrero* - Universidad Nacional Autónoma de México
}

\section{Resumen}

El presente trabajo forma parte de una investigación más amplia ${ }^{1}$, en la cual se busca analizar: 1) el impacto que el conocimiento generado y transferido hacia el sector acuícola tiene sobre el desarrollo regional y; 2) conocer en qué medida los procesos interactivos y la construcción de redes han dado lugar a la conformación de un capital social basado en conocimiento para el sector acuícola en el noroeste de México. El enfoque teórico-conceptual de esta investigación incorpora diversos planteamientos derivados de cuatro marcos analíticos: a) el de redes de conocimiento; b) el enfoque del capital social, c) el de los sistemas regionales de innovación $\mathrm{y}, \mathrm{d}$ ) aspectos relacionados a la idea de sociedades basadas en conocimiento. A través de una estrategia metodología basadas en estudio de casos, se analizan procesos específicos de generación y transferencia de conocimiento científico y tecnológico en el sector acuícola, cuando estos son resultado de interacciones entre diversos actores a nivel regional y local. En particular, se identifican las interacciones que son resultado de la colaboración que se promueven entre las instituciones académicas (centros, institutos e instituciones de educación superior), con otros sectores de la sociedad (gobierno, unidades productivas sociales y privadas).

Palabras clave: Redes de conocimiento, Sistemas regionales de innovación, Acuacultura en México.

\begin{abstract}
Abstract

This paper is part of a wider research, which attempts to analyze: 1) the impact that the knowledge generated and transferred over to the acqua-culture sector has on regional development and; 2 ) to learn to what degree the interactive processes and the creation of networks have given place to the conformation of a social capital based in knowledge for this sector in Mexico's North West. This research's theoretical-conceptual focus incorporates diverse approaches derived from four analytical frames. A) Knowledge networks; b) social capital; c) regional innovation systems and, d) aspects related to the idea of societies based on knowledge. Through a methodological strategy based on case study, specific processes for the generation and transfer of scientific and technological knowledge were analyzed within that sector, when these were the result of the interaction of diverse regional and local actors. Specifically, the identified interactions were those that resulted from the collaboration between academic institutions (Research Centers, Institutes or Higher Education Institutions) and others sectors (Government, units of social and private production).
\end{abstract}

Key Words: Knowledge Networks, Regional Innovation Systems, Water culture in Mexico.

\footnotetext{
*Investigadora Titular "C", del Instituto de Investigaciones Sociales, Universidad Nacional Autónoma de México. Enviar correspondencia a: (Dra. Rosalba Casas Guerrero rcasas@servidor.unam.mx).

${ }^{1}$ El proyecto más amplio se titula: “PyMES: Redes de conocimiento, actividad innovativa y desarrollo local", y está financiado por CONACYT. La responsable general es la Dra. Gabriela Dutrénit, profesorainvestigadora del Departamento de Producción Económica de la Universidad Autónoma Metropolitana Unidad Xochimilco.
} 
REDES- Revista hispana para el análisis de redes sociales

Vol.17,\#6, Diciembre 2009

http://revista-redes.rediris.es

\section{Introducción ${ }^{2}$}

El objetivo fundamental de este trabajo, es analizar la forma como se construyen redes para la producción y transferencia de conocimientos en el sector acuícola en la región noroeste de México, y las implicaciones que este proceso podría tener para el desarrollo de esta área geográfica del país.

La investigación parte del supuesto de que la capacidad de generación de conocimientos que posee el país, localizada principalmente en las universidades y centros de investigación, en las instituciones gubernamentales así como en los sectores productivos, son elementos importantes para impulsar el desarrollo económico y social a nivel regional y local.

El trabajo se divide en cuatro secciones o apartados. En la primera se expone el marco analítico de la investigación, el cual articula las nociones de aprendizaje interactivo, redes de conocimiento, capital social y sociedades basadas en conocimiento, así como la hipótesis de trabajo y la estrategia metodológica centrada en estudios de casos. En la segunda parte, se describe brevemente el desarrollo del sector acuícola en México, particularmente en la región noroeste (estados de Sinaloa, Sonora, Baja California y Baja California Sur), haciendo énfasis en sus capacidades locales $\mathrm{y} / \mathrm{o}$ regionales (disponibilidad de instituciones educativas, recursos humanos, centros e institutos de investigación, instituciones y programas de apoyo gubernamentales), todo lo cual conforma un espacio de conocimiento en el que se lleva a cabo la interacción, la transferencia de conocimiento y la construcción de capital social. En el tercer apartado, se analizan las interacciones entre los centros de investigación, las instituciones gubernamentales y las pequeñas y medianas empresas acuícolas. Finalmente, en la cuarta y última sección se reflexiona en torno al alcance y la naturaleza de las interacciones entre estos actores, destacando su importancia para la construcción de un capital social basado en conocimiento, susceptible de estimular el desarrollo regional y local.

\section{Marco analítico y estrategia metodológica}

El enfoque teórico-conceptual de esta investigación incorpora elementos derivados de tres analíticos: a) el de redes de conocimiento; b) el de capital social, y c) el enfoque de los sistemas regional y/o local de innovación. Adicionalmente, la

\footnotetext{
2 Una versión más desarrollada de este trabajo fue publicada en: Casas, R., J. Dettmer, L. Celis y C. Hernández, "Redes y Flujos de Conocimiento en la Acuacultura Mexicana", Revista REDES de Estudios Sociales de la Ciencia, Vol. 13, Num. 25, Universidad de Quilmes, Buenos Aires, pp. 111-144.
} 
REDES- Revista hispana para el análisis de redes sociales

Vol.17,\#6, Diciembre 2009

http: //revista-redes.rediris.es

construcción del enfoque teórico de esta investigación se apoya en los avances de trabajos anteriores que hemos realizado sobre esta temática (Casas y Luna, 1997; Casas, De Gortari y Santos, 2000; Casas, 2001; Luna, 2003).

\section{El enfoque de Redes de conocimiento}

La noción de redes sociales ha recibido aportes importantes de diversas disciplinas y marcos interpretativos (véanse por ejemplo, los trabajos de Mitchell, 1973; Knoke, 1990, Hedstrom y Swedberg, 1994), que son sugerentes para nuestro estudio.

La idea básica del enfoque de redes es que los individuos son actores intencionales, con motivaciones sociales y económicas, cuyas acciones están influenciadas por una red de relaciones en las cuales están insertos (Granovetter, 1984). Así, los actores y las relaciones que mantienen entre ellos, forman una red social, siendo un elemento clave la posición que cada actor ocupa en ella, lo que forma la estructura general de la red, la cual, a su vez, implica oportunidades y restricciones para los actores.

Existen varias nociones de redes las cuales se aplican a distintos niveles. A nivel micro, los analistas de la red examinan díadas, tríadas, otros subgrupos pequeños y redes centradas en ego. Tales estructuras son únicas a la perspectiva de redes. Al nivel macro la atención es a menudo puesta sobre la configuración de redes enteras y la identificación de posiciones estructurales y componentes de la red.

Entre los estudios de redes aplicados a la ciencia y la tecnología destacan los de redes sociotécnicas (Elzen, et al., 1996); redes tecnocientíficas; redes de innovación (De Bresson y Ámese, 1991) y, redes de conocimiento, centradas en la generación, transferencia y uso del conocimiento entre diferentes agentes (Gross, et al., 2001).

El concepto de redes de conocimiento tiene antecedentes en la noción de "ambiente regional de innovación", definido como el conjunto de instituciones académicas, industriales y políticas que -intencional o aleatoriamente- colaboran conjuntamente para impulsar las condiciones locales para la innovación (Etzkowitz y Uzzi, 1996).

Gross, et al. (2001: 7), definen la red conocimiento como "un conjunto de actividades emprendidas por actores autónomos discretos dotados con capacidad de consumir y producir conocimiento que incrementa el valor de las actividades de los actores, contribuye a la expansión del conocimiento, extendiendo el alcance para las aplicaciones de nuevo conocimiento". 
REDES- Revista hispana para el análisis de redes sociales

Vol.17,\#6, Diciembre 2009

http://revista-redes.rediris.es

Para estos autores, las redes de conocimiento son "estructuras especialmente difusas, a menudo agregaciones de individuos y organizaciones, vinculadas por medio de intereses compartidos e interesados acerca de un problema". Estos individuos y organizaciones son autónomos pero se unen para generar y añadir conocimiento acerca de problemas compartidos. Las redes de conocimiento generalmente no tienen fronteras rígidamente definidas y comparten conocimiento más allá de fronteras políticas y sociales (Gross et al., 2001: 7-8).

De acuerdo con estos autores, las redes de conocimiento operan crecientemente dentro de un contexto de aplicación: los problemas son puestos dentro de un marco de trabajo transdisciplinario más bien que unidisciplinario, y la investigación se lleva a cabo en formas organizadas heterogéneamente, no jerárquicas, que son esencialmente transitorias más que permanentes. Siguiendo a Gibbons (1994), estas redes pueden ser vistas como esquemas de producción de conocimiento socialmente distribuido donde el conocimiento es diseminado por aquellos quienes son sus productores activos. Por lo tanto, en el proceso de conocimiento las redes tienen el potencial para contribuir a la definición de problemas, la formación de la agenda de investigación, la ejecución de la investigación y la diseminación de los resultados. Desde esta óptica, las redes de conocimiento conducidas por la universidad proveen una base ideal para una evaluación preliminar de si y dónde las redes contribuyen, en la cadena de generación, producción, y diseminación del conocimiento (Gross, 2001: 9).

En este trabajo el concepto de redes de conocimiento se aplica al análisis de las relaciones entre los diferentes actores que intervienen en el proceso de generación e intercambio de conocimientos (Casas, 2003). Desde nuestra perspectiva estas redes se construyen mediante intercambios entre un conjunto de actores que tienen intereses comunes en el desarrollo o aplicación del conocimiento tradicional, científico, tecnológico o técnico para un propósito específico, sea éste científico, de desarrollo tecnológico o de mejoramiento de procesos productivos. Estas formas de intercambio pueden concebirse como un proceso de transacción (Mitchell, 1973) de conocimiento, aunque no en términos económicos, ya que una gran parte del conocimiento que se transmite en estas redes es tácito y no se efectúa mediante la compra-venta del mismo.

\section{El enfoque de capital social}

El uso del concepto de capital social se remonta a los inicios del siglo XX, cuando fue aplicado por Hanifan para analizar el papel de las comunidades en la satisfacción de necesidades sociales de los individuos. 
REDES- Revista hispana para el análisis de redes sociales

Vol.17,\#6, Diciembre 2009

http://revista-redes.rediris.es

Si bien durante muchos años la idea de capital social permaneció en el olvido, en las últimas décadas del siglo $\mathrm{xx}$, el concepto fue recuperado por autores como Bordieu y Coleman, quienes le dieron nuevas dimensiones. ${ }^{3}$ En años recientes se han desarrollado una gran cantidad de definiciones de capital social, las cuales difieren en función de la unidad de análisis y el tipo de problemas que pretenden explicar. Putnam (2003), por ejemplo, define el capital social como las “características de las organizaciones sociales, tales como confianza, normas y redes, que pueden mejorar la eficiencia de la sociedad por medio de facilitar acciones coordinadas". Por su parte, Nahapiet y Ghoshal, (1998: 243), entienden el capital social como "la suma de recursos reales y potenciales incorporados disponibles a través, y derivados de redes de relaciones poseídas por un individuo o unidad social. El capital social por lo tanto, comprende las redes y las ventajas que podrían ser movilizadas a través de esa red". En su estudio para el Banco Mundial, Grootaert y van Bastelaer (2002) definen el capital social como "instituciones, relaciones, actitudes y valores, que gobiernan las interacciones entre la gente y contribuye al desarrollo económico y social". Finalmente, Fountain (1998: 105) afirma que, parecido a otras formas de capital (físico y humano), el "capital social" se refiere a las características de la organización social, tales como redes, normas y confianza que facilitan la coordinación y la cooperación para beneficio mutuo.

Pese a sus distintas conceptualizaciones, la idea central de la teoría del capital social es que las redes sociales son importantes porque poseen un valor, (sobre todo para quienes se encuentran en ellas) y están orientadas a la resolución de problemas. Se asume que las acciones de los individuos y grupos pueden ser grandemente facilitadas por su membresía en redes sociales, especialmente por sus vínculos directos e indirectos con otros actores en estas redes. Bajo esta perspectiva, el capital social es útil para explicar el éxito diferencial de individuos y firmas en su rivalidad competitiva (Adler y Kwon, 2000: 90).

Desde la óptica de las organizaciones basadas en conocimiento, el capital social es considerado un facilitador para adquirir, compartir, transferir y utilizar conocimiento codificado y tácito. Dado que éste último es más difícil de obtener, los individuos y las firmas deben ser capaces de identificar a otros (individuos y firmas) con

3 Bourdieu, definió el capital social como "la acumulación de recursos reales y potenciales ligados a la posesión de una red duradera de relaciones más o menos institucionalizadas de mutua familiaridad y reconocimiento... que provee para cada uno de sus miembros el soporte de capital de propiedad colectiva" (Bourdieu, 1977). Por su parte Coleman extendió el concepto hasta abarcar dimensiones macro, considerando el capital social como "una variedad de diferentes entidades con dos elementos en común: todas consisten en algún aspecto de las estructuras sociales y facilitan cierta acción de los actores - ya se trate de personas o actores corporativos- dentro de la estructura" (Coleman, 2000: 16). 
REDES- Revista hispana para el análisis de redes sociales

Vol.17,\#6, Diciembre 2009

http://revista-redes.rediris.es

expertise; construir relaciones que hagan atractivo el compartir conocimiento con otros individuos u organizaciones y desarrollar un lenguaje compartido que les facilite absorber y utilizar el conocimiento. En este contexto, la existencia de un intermediario o traductor (broker), que salve la distancia entre quienes poseen la información o el conocimiento y quienes no lo tienen, es fundamental para incrementar el nivel de capital social.

Cuando las organizaciones adquieren formas complejas (por ejemplo, redes entre firmas, alianzas, clusters o distritos industriales), el papel del capital social en la transferencia de conocimiento es muy importante, pero el peso de factores tales como la confianza, la credibilidad, un lenguaje común, una cultura compartida, motivación, etc., dependen mucho de las características estructurales, relacionales y cognitivas de la red, así como del contexto económico, social y geográfico considerado.

Es importante destacar que el capital social no conduce automáticamente a la transferencia de conocimiento para el bienestar económico y social y, que por contrario, ciertas formas de capital social -por ejemplo, los monopolios o los carteles- pueden tener consecuencias indeseables para la sociedad. En estas condiciones, como señala Fountain (1998), es necesario tener presente que el capital social constituye sólo uno de entre muchos factores, que a la vez que facilita la cooperación entre individuos y organizaciones, propicia el intercambio de información, estimula la competencia, procura las ganancias compartidas y alienta la confianza para asegurar reciprocidad y el "juego limpio" dentro de una red.

La significación del capital social para la innovación y para las políticas de ciencia y tecnología parece quedar fuera de todo duda cuando se observan el impacto que las redes de individuos, grupos y organizaciones basados en el aprendizaje y la transferencia de conocimiento tienen para desarrollo económico y social de los países y regiones como la que aquí estudiamos. En este sentido, consideramos que la confianza, la reciprocidad, las redes, y un lenguaje compartido, constituyen elementos importantes para conformación de un capital social capaz potenciar el desarrollo económico a nivel regional y local.

\section{Enfoque regional/ local}

En el análisis de los procesos de generación, transferencia y flujos del conocimiento, el enfoque de los sistemas de innovación reviste actualmente gran importancia, ya que se ha atribuido a las regiones un papel dinámico, concibiéndolas como un importante motor del desarrollo. 
REDES- Revista hispana para el análisis de redes sociales

Vol.17,\#6, Diciembre 2009

http://revista-redes.rediris.es

El punto de partida de estos trabajos se ha centrado en la adopción de una nueva concepción de la innovación basada en la teoría económica evolucionista (Nelson y Winter, 1982). Desde esta perspectiva, la innovación consiste en un proceso de aprendizaje entre actores que descansa en el conocimiento tácito y las habilidades. Se trata de procesos de aprendizaje enraizados en la sociedad y el territorio, a través de los cuales se intercambian y transfieren conocimientos codificados y tácitos, que se difunden como consecuencia de las relaciones entre los actores.

Según Etemad y Chu (2004: 48), la “idea de que la innovación es un proceso colectivo, es además iluminada por el llamado paradigma de red" de la innovación (Hakansson, 1987; Lundval, 1992). El paradigma de red propone que la innovación es fundamentalmente un proceso interactivo (Rosenberg, 1992; Von Hippel, 1988). Es decir, las interacciones que buscan la innovación involucran "aprendizaje interactivo entre los agentes: entre áreas funcionales dentro de la firma (relaciones entre firmas); entre usuarios y productores, y entre firmas e instituciones, apoyando el desarrollo de la región". Son estas redes las que facilitan el intercambio de información, conocimientos $y$, en general, recursos del sistema productivo.

Esta perspectiva se ha visto enriquecida por el enfoque de los "sistemas de innovación". Como se sabe, Christopher Freeman (1987) fue el primero en definir el concepto de sistema de innovación, entendido como una "red de instituciones en los sectores público y privado cuyas actividades e interacciones inician, importan, modifican y difunden nuevas tecnologías". Por su parte, Lundvall (1992) hizo una distinción entre una definición estrecha y una amplia de sistema [nacional] de innovación. Su definición estrecha de sistema de innovación incluye "organizaciones e instituciones implicadas en la búsqueda y exploración - tales como departamentos de Investigación y Desarrollo (I\&D), institutos tecnológicos y universidades-." En su definición extensa, un sistema de innovación incluye "todas las partes y aspectos de la estructura económica y la institucional establecida afectando el aprendizaje así como también buscando y explorando el sistema de producción en el cual el aprendizaje toma lugar".

Como resultado de diversas revisiones críticas e investigaciones empíricas, esta visión limitada nacionalmente -cuando menos en términos geográficos-, fue relativizada entre otros, por los geógrafos economistas quienes, al tratar de explicar las relaciones entre conocimiento local y éxito económico, a través de conceptos tales como innovative melieu e industrial district, hicieron cada vez más énfasis en la importancia de las regiones. De esta forma, el enfoque de los sistemas 
REDES- Revista hispana para el análisis de redes sociales

Vol.17,\#6, Diciembre 2009

http://revista-redes.rediris.es

de innovación fue ampliado para incluir sistemas de innovación sectoriales y otros situados a diferente escala geográfica. En este contexto, en los últimos lustros, la noción de sistemas regionales de innovación ha ido emergiendo como una perspectiva de análisis territorialmente localizada, deriva del concepto más amplio de sistema nacional de innovación.

Puesto que la idea de sistema regional de innovación fue inspirada en parte, por las teorías de la aglomeración dentro de la ciencia regional y la geografía económica, así como por el éxito de los clusters regionales y los distritos industriales, algunos han tendido a conceptualizar un sistema regional de innovación como un cluster regional rodeado por organizaciones que soportan el conocimiento. En este sentido, Asheim e Isaksen (2002), afirman: "Las regiones son vistas como importantes bases de coordinación económica y governanza al nivel meso entre lo nacional y lo local (cluster y firmas)". En otras palabras, "la región es crecientemente el nivel al cual la innovación es producida a través de redes regionales de innovadores, clusters locales y los efectos de fertilización cruzada de las instituciones de investigación" (Lundvall y Borrás, 1997).

Por su importancia para analizar y documentar la interacción y el flujo de conocimientos entre diversos actores, en nuestro trabajo asignamos un lugar especial a espacios geográficos como las regiones y localidades en la creación de redes y flujos de conocimiento. Partimos de un concepto de región concebida como una entidad que cuenta con un conjunto de características favorables para la construcción de redes de conocimiento, tales como capacidades de investigación, sectores productivos sensibilizados sobre la importancia de estas actividades, políticas regionales, estatales y/o locales en este campo, y la formación de agentes mixtos para promover las interacciones.

Las redes de conocimiento a nivel regional y/o local se constituyen en la unidad de análisis de esta investigación. A partir de los estudios realizados en diversos campos tecnológicos y de proyectos específicos, sostenemos que, mediante las redes de conocimiento, es posible contribuir a la formación de espacios locales y/o regionales de conocimiento (Casas, De Gortari y Santos, 2000; Casas, 2001; Casas, Luna y Santos, 2001: 359).

\section{Estrategia metodológica}

Con base en los planteamientos teóricos anteriores, sostenemos la hipótesis de que las interacciones entre las instituciones académicas, el gobierno y los sectores productivos, parecen estar sustentadas en procesos de aprendizaje, generación de confianza, normas de reciprocidad y la existencia de redes, los cuales, bajo 
REDES- Revista hispana para el análisis de redes sociales

Vol.17,\#6, Diciembre 2009

http: //revista-redes.rediris.es

determinadas condiciones, pueden contribuir a la formación de un capital social basado en conocimiento. Si esto fuese así, entonces dicho capital social podría jugar un papel importante en la formación y/o consolidación de sistemas regionales y/o locales de innovación.

Para contrastar esta hipótesis, se adoptó una estrategia metodológica basada en estudios de casos. El estudio de caso permitió combinar varias fuentes de evidencia. Así, la información recogida a través de las entrevistas a profundidad fue complementada con otras provenientes de fuentes documentales, tales como planes oficiales de desarrollo del sector, anuarios estadísticos, directorios de organismos públicos, padrones de empresas acuícolas e investigaciones académicas nacionales e internacionales publicadas. En total se realizaron 81 entrevistas a profundidad en la región noroeste. El análisis que presentamos aquí, se basa principalmente en 31 entrevistas, realizadas a un conjunto de 28 pequeñas y medianas empresas acuícolas (sociales y privadas).

Entre las instituciones académicas entrevistadas cabe mencionar: el Centro de Investigaciones Biológicas del Noroeste (CIBNOR), el Centro de Investigaciones en Alimentación y Desarrollo (CIAD-Mazatlán), el Centro de Ciencias de Sinaloa (CCS), el Departamento de Investigaciones Científicas y Tecnológicas de la Universidad de Sonora (DICTUS-UNISON), la Facultad de Ciencias Marinas y el Instituto de Investigaciones Oceanológicas de la Universidad Autónoma de Baja California, así como el Centro de Investigación Científica y Educación Superior de Ensenada (CICESE). Algunas de las instituciones de gobierno visitadas fueron: las Subdelegaciones de Pesca y Acuacultura de la SAGARPA-CONAPESCA, dependencias estatales donde se encuentran las Direcciones de Acuacultura, Institutos de Acuacultura y Consejos Estatales de Ciencia y Tecnología.

El foco de las entrevistas se centró en las colaboraciones e interacciones existentes entre diferentes actores (universidades, centros de investigación, dependencias de gobierno y empresas acuícolas privadas así como asociaciones de productores y/o cooperativas). Adicionalmente, se buscó conocer qué ocurre en el proceso de construcción de redes entre estos actores, cómo influye esto en los procesos de generación, distribución y uso de conocimiento, los resultados que se obtienen de estas colaboraciones y los alcances de las mismas para desarrollar una acuacultura basada en conocimiento en la región. En el tercer apartado presentamos un análisis preliminar de la información recabada. 
REDES- Revista hispana para el análisis de redes sociales

Vol.17,\#6, Diciembre 2009

http: // revista-redes.rediris.es

\section{La acuacultura en la región noroeste}

La acuacultura consiste en la producción de plantas o animales acuáticos en sistemas controlados donde su crecimiento es manejado o mejorado por el hombre. Los principales organismos cultivados son peces, moluscos y crustáceos, pero otras especies también se cultivan en menores cantidades.

La acuacultura en México se ha desarrollado en todas las regiones del país, usando prácticas diversas, extensivas con sistemas abiertos y cerrados, así como intensivas y semi-intensivas, en jaulas, encierros, líneas suspendidas, estanques artesanales, canales de corriente rápida, estanques de concreto, cubiertas plásticas y otras tecnologías disponibles para criar especies acuáticas para el autoconsumo o comercio de productos.

El Noroeste de México es considerado como la región de mayor productividad biológica del país, debido a una combinación de procesos oceánicos que favorecen una alta productividad primaria, básica para el sostenimiento de recursos masivos, y la existencia de ecosistemas diversos que propician la presencia de una gran diversidad de especies (Lluch y Hernández, 2006: 107).

En esta región se han identificado alrededor de 650 especies que pueden ser utilizadas para consumo humano e industrialización, de las cuales sólo unas pocas son objeto de explotación, bien por sus grandes volúmenes (como los pelágicos menores o el calamar); bien por su alto valor en el mercado (como el camarón, la langosta y el abulón). Por lo tanto, en la región existe un gran potencial pesquero y acuícola (Avilés y Vázquez, 2006: 73).

Para la acuacultura, la región noroeste es la más importante del país, ya que cerca del $65 \%$ de la producción nacional proviene de esta zona. Sonora y Sinaloa aportan cerca del $40 \%$ de la producción total con más de 300 granjas de cultivo de camarón blanco (litopenaus vannamei), mientras que los principales laboratorios de producción de poslarvas de camarón se encuentran en Baja California Sur, entidad que también dispone de especies marinas de alto valor comercial como la cabrilla, el pargo rojo, el atún aleta amarilla, el robalo y el lenguado, los cuales son susceptibles de cultivo. Adicionalmente la región es reconocida por sus moluscos de alto valor comercial, como la almeja mano de león, las ostras perleras, el abulón y el callo de hacha (Avilés y Vázquez, 2006: 73). 
REDES- Revista hispana para el análisis de redes sociales

Vol.17,\#6, Diciembre 2009

http://revista-redes.rediris.es

Actualmente, la región noroeste es la más importante del país en términos de capacidad de procesamiento de productos pesqueros. Asimismo, cuenta con el menor número de habitantes con respecto a otras regiones, con el mayor porcentaje territorial, el menor porcentaje de PIB, ocupa la tercera posición en posgrados aprobados y dispone de un importante número de investigadores adscritos a centros públicos de investigación tales como el CIBNOR, el CIAD, así como otras instituciones como el Instituto Nacional de Pesca (INP), el Centro de Investigaciones y Ciencias Marinas del Instituto Politécnico Nacional (CICIMAR-IPN), la Universidad Autónoma de Sinaloa (UAS), la Universidad de Sonora (UNISON) y la Universidad Autónoma de Baja California (UABC), entre otras. Con todo, la acuacultura de la región enfrenta problemas de diversa naturaleza, entre los que destacan: patologías en la camaronicultura y otras especies; falta de capacitación en sanidad e inocuidad alimentaria; desplazamiento de productos mexicanos de los mercados de Estados Unidos y Europa, por productos asiáticos; falta de capacitación de los trabajadores; creciente importación de tecnologías, equipo e insumos extranjeros; insuficiente vinculación de los institutos de investigación y las universidades con las PYMES del sector e incipientes esfuerzos de integración de los productores acuícolas y comercializadores para formar empresas integradoras, alianza o clusters.

\section{Actores, redes de conocimiento y procesos interactivos en la región noroeste}

La investigación permitió identificar un conjunto de actores institucionales, tanto de carácter académico como gubernamental, cuyo propósito es atender diversas necesidades y/o demandas del sector acuícola en las cuatro entidades federativas estudiadas que abarcan la región, así como la ejecución de una serie programas para impulsar la generación, transferencia y aplicación de conocimiento en la acuacultura. Distinguimos tres actores fundamentales: 1) Instituciones gubernamentales (tales como Subdelegaciones de Pesca y Acuacultura de la SAGARPA-CONAPESCA); 2) Instituciones académicas, y 3) unidades productivas acuícolas, privadas y sociales.

De igual forma, en el trabajo nos centramos en 5 tipos de interacciones que se dan entre estos tres actores: 1) academia y gobierno, 2) academia y empresa, 3) gobierno y empresa, 4) empresa-empresa y 5) academia-empresa-gobierno.

La interacción tipo 1, academia-gobierno, se da casi de forma insoslayable, ya que tanto la formación de recursos humanos de alto nivel como la investigación acuícola en México se financia principalmente por el gobierno federal, a través de CONACYT, y 
REDES- Revista hispana para el análisis de redes sociales

Vol.17,\#6, Diciembre 2009

http://revista-redes.rediris.es

en menor grado por la SAGARPA-CONAPESCA. Existe también, en algunos casos, financiamiento por parte de los gobiernos estatales.

El tipo 2, academia-empresa, es un modo de interacción que no se ha manifestado como necesario para cada uno de estos dos actores, debido a que sus actividades se han desarrollado casi de manera independiente.

El tipo 3, gobierno-empresa, puede considerarse una relación necesaria y obligatoria en el sector acuícola, ya que las empresas acuícolas requieren la expedición por parte del gobierno de permisos de operación y cosecha, entre otros trámites.

Por lo que se refiere a las interacciones del tipo 4, empresa-empresa, las definimos como aquellas que se dan entre las diferentes empresas acuícolas, ya sea como productoras, proveedores de insumos y alimentos y comercializadoras del producto.

Finalmente, las interacciones tipo 5, las concebimos como aquellas que involucran tanto a los actores del sector académico, como del productivo y el gobierno.

A continuación presentamos un análisis de las interacciones que hemos identificado entre los distintos actores y que parecen estar dando lugar a la formación de redes de conocimiento, a partir de la información proporcionada por las personas entrevistadas. Para ello, clasificamos la información obtenida de 31 entrevistas en ocho grandes ejes o núcleos temáticos que incluyen las principales motivaciones para la interacción:

Asesoría y capacitación

Investigación y Desarrollo

Gestión

Incorporación de recursos humanos

Financiamiento

Abastecimiento, comercialización y venta

Provisión de servicios

Intercambio de información

\section{Breve caracterización de las empresas entrevistadas}

En la investigación de campo se entrevistó a un total de 28 empresas acuícolas, distribuidas de la siguiente manera: dos fueron microempresas, 12 fueron pequeñas empresas, diez medianas y cuatro grandes empresas (véase gráfica 1 ). 
REDES- Revista hispana para el análisis de redes sociales

Vol.17,\#6, Diciembre 2009

http://revista-redes.rediris.es

Del total las empresas acuícolas entrevistadas, seis fueron empresas sociales y 22 fueron empresas privadas (gráfica 2 ).

Gráfica 1. Número de empresas entrevistadas

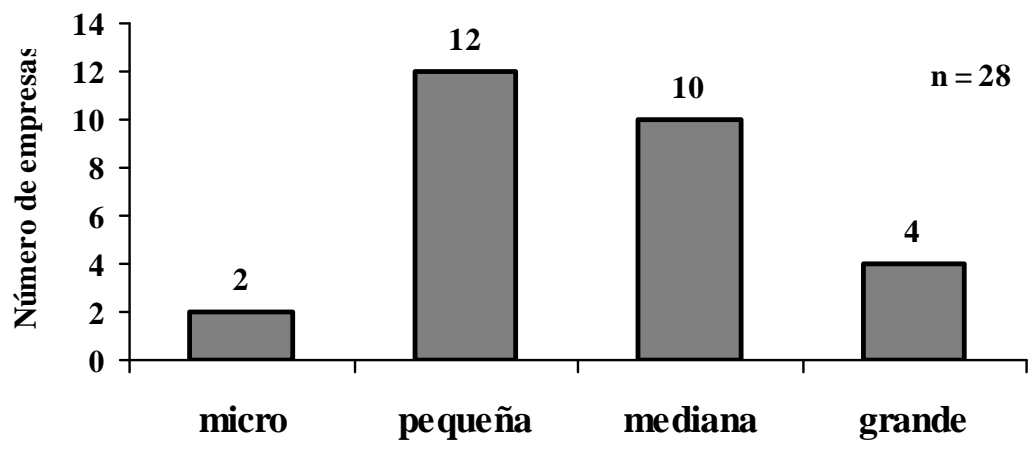

Fuente: Entrevistas a PyMES acuícolas de la región Noroeste de México.

Gráfica 2. Razón social de las empresas entrevistadas

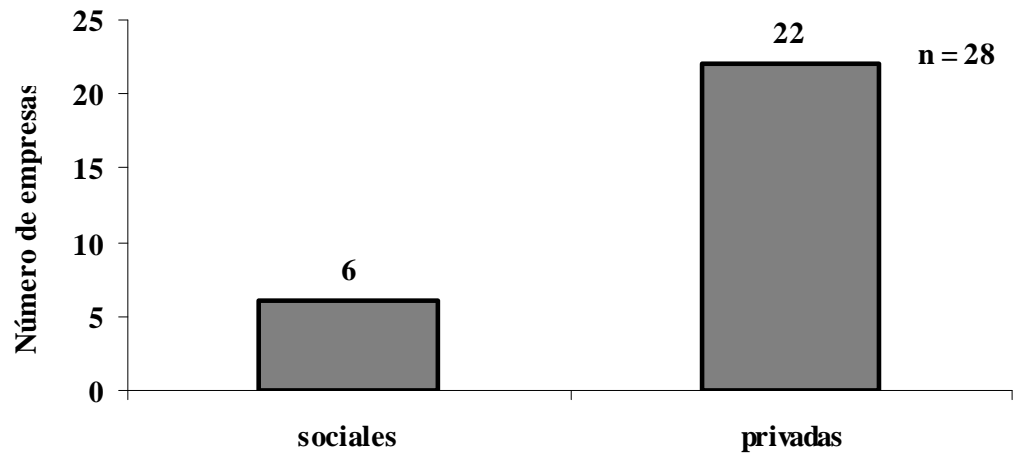

Fuente: Entrevistas a PyMES acuícolas de la región Noroeste de México.

Tomando en cuenta la características de las empresas por tipo de cultivo y etapa en la cadena productiva, encontramos que 18 empresas se dedicaban exclusivamente a la engorda, tres a la producción de semilla, y siete desarrollaban ambos procesos de la cadena productiva (véase gráfica 3 ). Finalmente, del total de las empresas entrevistadas, 12 se orientaban al mercado nacional, tres a la exportación y 13 a ambos tipos de mercado (gráfica 4). 
REDES- Revista hispana para el análisis de redes sociales

Vol.17,\#6, Diciembre 2009

http://revista-redes.rediris.es

Gráfica 3. Empresas entrevistadas por cultivo y etapa de la cadena productiva

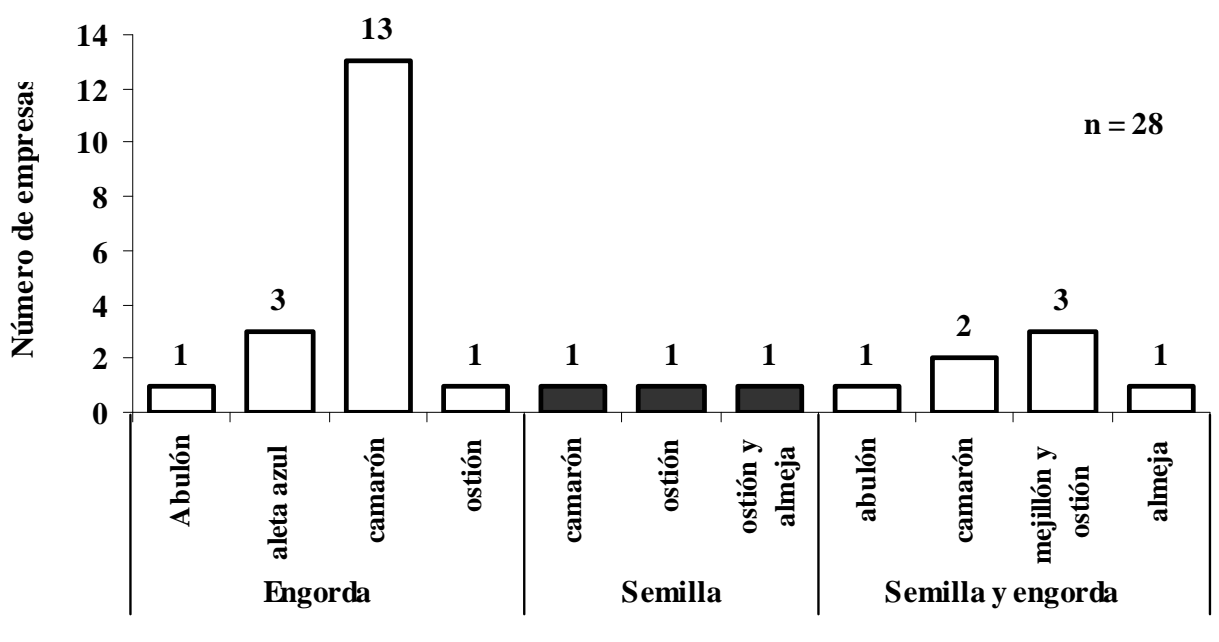

Fuente: Entrevistas a PyMES acuícolas de la región Noroeste de México.

Gráfica 4. Mercados de las empresas entrevistadas

$\mathbf{n}=\mathbf{2 8}$

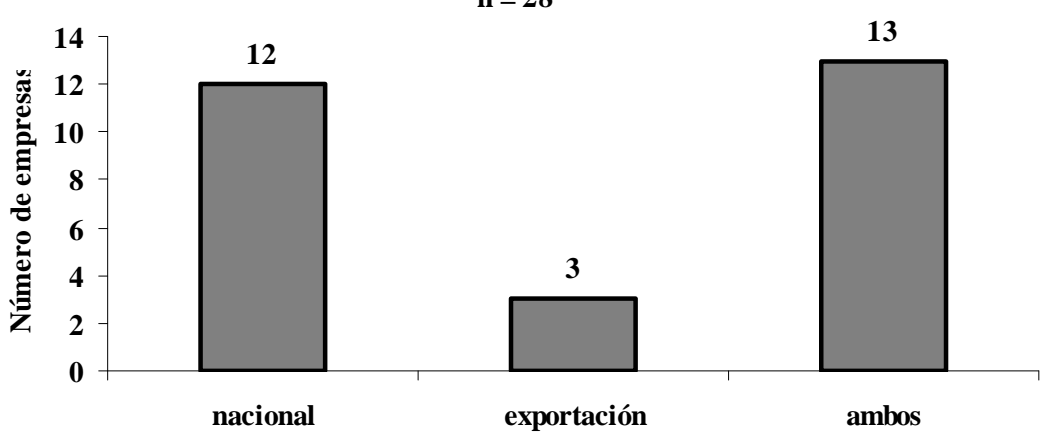

Fuente: Entrevistas a PyMES acuícolas de la región Noroeste de México.

\section{Interacciones tipo 1, academia-gobierno}

La información recabada de las entrevistas y otras fuentes documentales sugiere que, en general, existe un buen nivel de interacción entre ambos actores para resolver problemas y apoyar el desarrollo de acuacultura en la región. Entre las principales instituciones de formación de recursos humanos e investigación que mantienen relaciones con dependencias de los gobiernos federal y estatales destacan: el CIBNOR, el CIAD, el Centro de Investigación Científica y Educación Superior de Ensenada (CICESE), el Instituto de Ciencias del Mar y Limnología (ICMLUNAM), el Centro de Investigación en Ciencias del Mar (CICIMAR-IPN) y las Universidades Autónoma de Sinaloa, de Sonora y Baja California, entre otras. 
REDES- Revista hispana para el análisis de redes sociales

Vol.17,\#6, Diciembre 2009

http: //revista-redes.rediris.es

Se trata de una red de centros que realizan proyectos de investigación básica y aplicada, comparten posgrados y estimulan la movilidad de sus investigadores (incluso hacia organismos públicos), casi siempre con apoyo de instancias de gobierno, lo que contribuye a la transferencia y flujos de conocimiento en el campo de los cultivos marinos, los recursos naturales y el impacto ambiental, para la conservación y el desarrollo sustentable de la región noroeste.

\section{I nteracciones tipo 2, academia-empresa}

Como puede observarse en la gráfica 5, entre las principales motivaciones de las pequeñas y medianas empresas (PYMES) acuícolas para interactuar con las instituciones académicas se encuentran la prestación de servicios, asesoría y capacitación, investigación y desarrollo, y recursos humanos para puestos de carácter técnico y administrativo. Lo anterior se reitera en las gráficas 6 y 7, las cuales muestran que, para muchas empresas, las instituciones académicas constituyen las principales proveedoras de recursos humanos.

Gráfica 5. Motivos de las relaciones de las PyMES con Instituciones Académicas

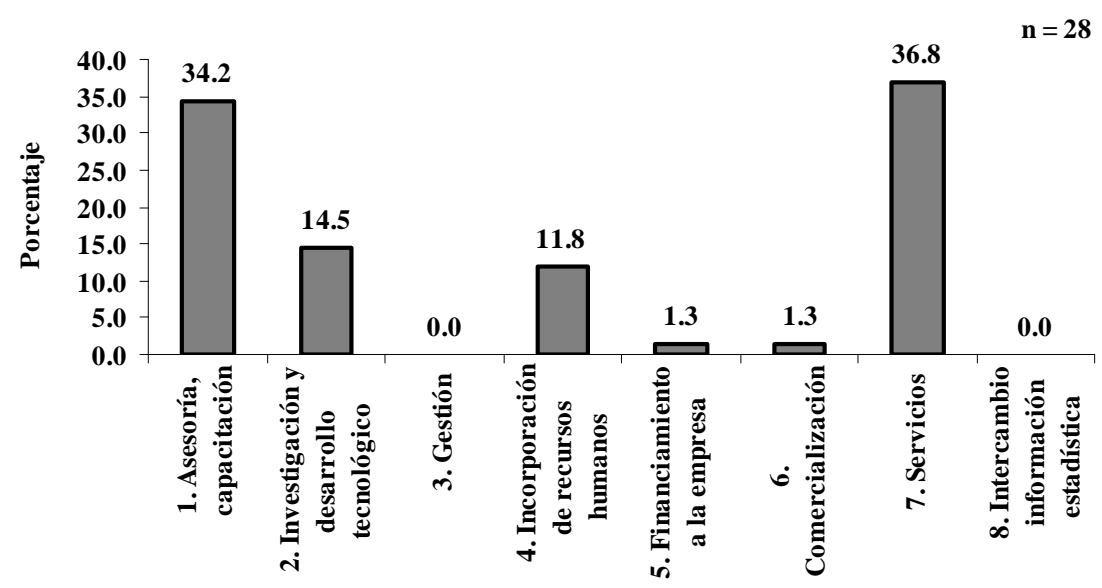

Fuente: Entrevistas a PyMES acuícolas de la región Noroeste de México. 
REDES- Revista hispana para el análisis de redes sociales

Vol.17,\#6, Diciembre 2009

http://revista-redes.rediris.es

Gráfica 6. Interacciones que las PyMES tienen con actores diversos para incorporación de recursos humanos

$\mathbf{n}=\mathbf{1 9}$

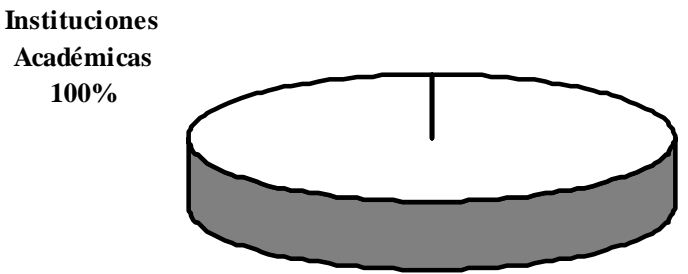

Fuente: Entrevistas a PyMES acuícolas de la región Noroeste de México.

Gráfica 7. Interacciones que las PyMES tienen con actores diversos para as esoría y capacitación

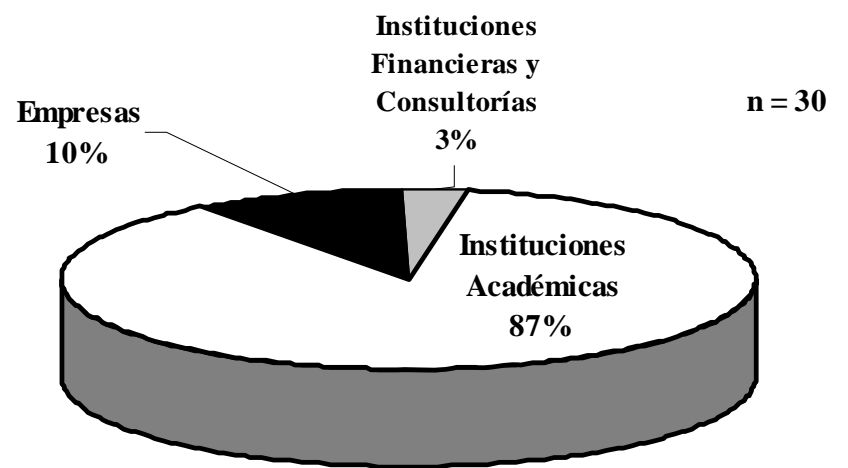

Fuente: Entrevistas a PyMES acuícolas de la región Noroeste de México.

Asimismo, la gráfica 8 muestra que, para fines de investigación, transferencia y adaptación de tecnología, las empresas acuícolas buscan interactuar en primer lugar con las instituciones académicas, en segundo término con otras empresas y en tercero con instituciones de gobierno. Lo anterior es importante porque tradicionalmente las instituciones académicas han tenido poco contacto con las empresas acuícolas para realizar investigación. Las entrevistas sugieren que la mayor parte de estas interacciones tienden a producirse generalmente en espacios tales como congresos de acuacultura, simposios, talleres, y revistas técnicas de acuacultura (como Panorama Acuícola Magazine e Industria Acuícola). 
REDES- Revista hispana para el análisis de redes sociales

Vol.17,\#6, Diciembre 2009

http://revista-redes.rediris.es

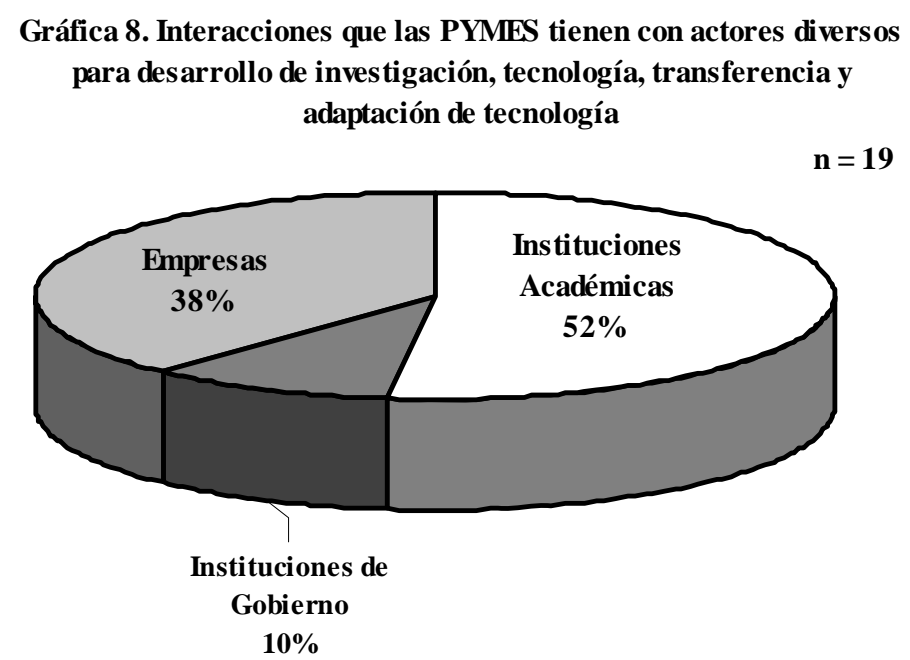

Fuente: Entrevistas a PyMES acuícolas de la región Noroeste de México.

Es en estos ámbitos donde empresarios y académicos entran en contacto y donde se comienzan a gestar las primeras interacciones. Generalmente se trata de relaciones informales, cara a cara (productor-investigador), basadas en la confianza que el empresario tiene en el conocimiento del investigador. Aunque generalmente se asume que la estructura de las instituciones académicas es fundamental para canalizar las demandas de las empresas, en el caso de la acuacultura parece ser que la confianza y disposición a la colaboración entre empresarios e investigadores es el factor más importante, como se ha documentado en otros trabajos (Luna, 2003).

De la información recabada a través de las entrevistas se puede inferir la construcción de redes y flujos de conocimientos incipientes, las cuales se han dado principalmente para satisfacer necesidades muy concretas, tales como: sugerencias técnicas de los investigadores; formación de recursos humanos para las empresas; servicios de análisis de agua (microbiológicos) y diagnósticos sanitarios y patológicos; mejora genética; asesoría en técnicas de producción; estudios de impacto ambiental; monitoreo de mareas rojas; nutrición, asesoría en plantas de alimentos; diversificación de cultivos; instalaciones para apoyar con cría de larvas y juveniles; préstamo de reproductores y asesoría en la elaboración de proyectos acuícolas.

Estas interacciones de carácter bilateral, han tenido, en general propósitos muy puntuales y no son muy duraderas ni estrechas en la mayoría de los casos. De la información recabada en las empresas, no se puede deducir que de estas interacciones hayan surgido nuevos desarrollos o innovaciones tecnológicas. 
REDES- Revista hispana para el análisis de redes sociales

Vol.17,\#6, Diciembre 2009

http://revista-redes.rediris.es

\section{Interacciones tipo 3, gobierno-empresa}

Tradicionalmente, el gobierno ha jugado un papel importante en el impulso del sector acuícola. En años recientes, pese a la creciente participación del sector privado en esta actividad, tanto el Gobierno Federal como los de los estados continúan coadyuvando significativamente a su desarrollo. La información obtenida de las entrevistas que se muestra en la gráfica 9 , indica que los requerimientos de gestión de las empresas son satisfechos principalmente por las instituciones de gobierno (federal o estatal) y en segundo término, por otras empresas. Especialmente en lo relativo a financiamiento, la gráfica 10 muestra que, para satisfacer sus necesidades de financiamiento, las empresas interactúan más frecuentemente con instituciones gubernamentales; en segundo término, con otras empresas $y$, en tercer lugar, con instituciones financieras que han sido creadas para este fin.

Gráfica 9. Interacciones que las PyMES tienen con actores diversos para gestionar permisos de operación, importación, exportación, apoyos económicos para fomento, investigación, etc.

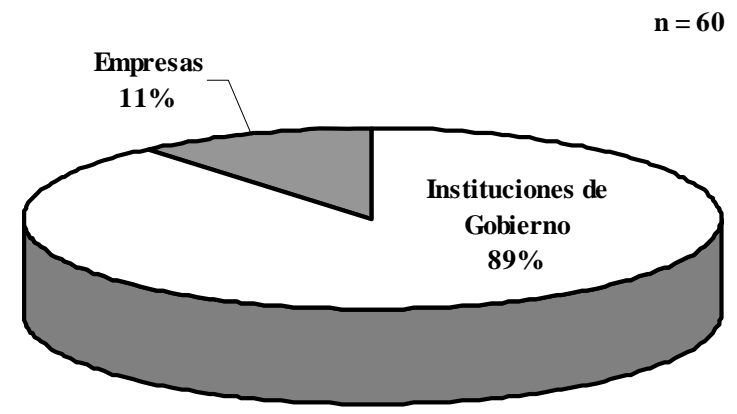

Fuente: Entrevistas a PyMES acuícolas de la región Noroeste de México. 
REDES- Revista hispana para el análisis de redes sociales

Vol.17,\#6, Diciembre 2009

http: //revista-redes.rediris.es

Gráfica 10. Motivos de las relaciones de las PyMES con

Instituciones de Gobierno

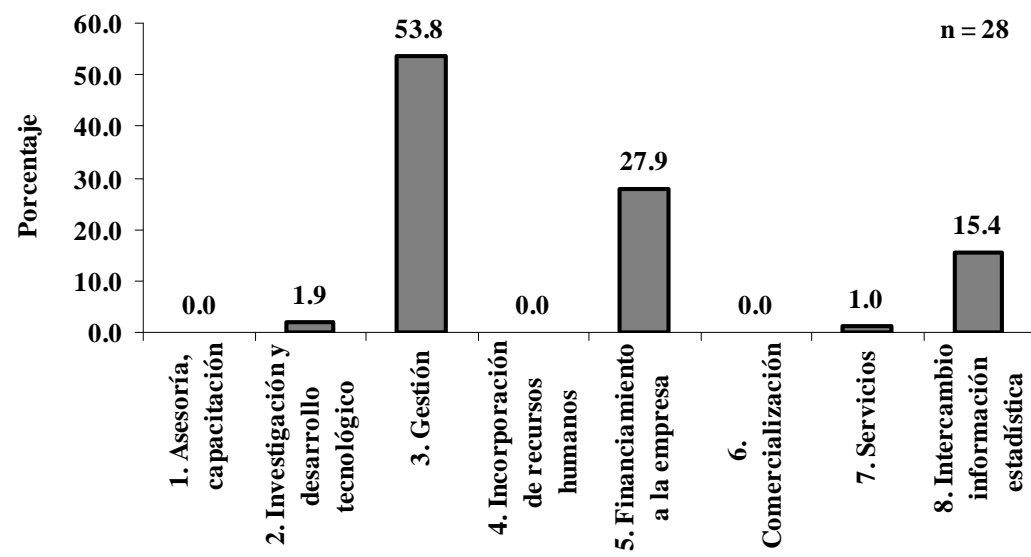

Fuente: Entrevistas a PyMES acuícolas de la región Noroeste de México.

Entre los programas de financiamiento que ofrecen las instituciones gubernamentales, los entrevistados mencionan: Alianza Contigo (SAGARPA), que otorga créditos para la puesta en marcha y mejora de la producción, proyectos de investigación, desarrollo tecnológico. El Fondo PYME de la Secretaría de Economía; FIRCO, para el equipamiento de laboratorios (estanques, tuberías, etc.); PRONAR, que otorga financiamiento para la capacitación técnica y proyectos demostrativos de tecnologías, para sectores de bajos ingresos y comunidades marginadas, y el Programa para la Construcción y Fortalecimiento de Redes de Valor, aplicado a especies prioritarias y la creación de Comités Sistema-Producto en diversas entidades federativas (SAGARPA).

\section{Interacciones tipo 4, empresa-empresa}

En cuanto a las interacciones tipo 4 (empresa-empresa), éstas presentan características distintas dependiendo de la naturaleza social o privado de la unidad productiva. En el primer caso, la asociación y el agrupamiento resultan indispensables para que estas empresas puedan sobrevivir. En el sector privado las interacciones son más bien escasas. Las entrevistas mostraron que entre las empresas privadas la falta de confianza tiende a debilitar la colaboración, aunque desde luego, se dan excepciones. Por ejemplo, en la gráfica 11 se observa que, para el abastecimiento y venta de productos, las PYMES acuícolas de la región establecen principalmente interacciones con otras empresas. Indagando sobre motivos para la interacción entre empresas, la gráfica 12 muestra que entre los principales sobresalen: la comercialización, el financiamiento, la investigación y desarrollo tecnológico y la gestión. 
REDES- Revista hispana para el análisis de redes sociales

Vol.17,\#6, Diciembre 2009

http://revista-redes.rediris.es

Gráfica 11. Interacciones que las PyMES tienen con actores diversos para el abastecimiento de insumos, equipo y la venta de los productos

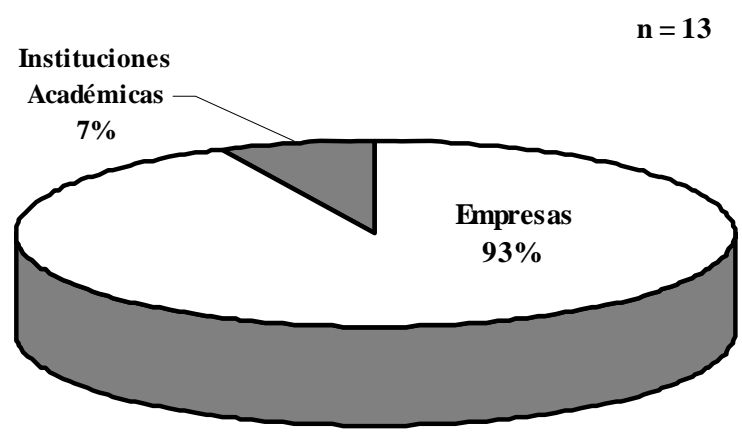

Fuente: Entrevistas a PyMES acuícolas de la región Noroeste de México.

Gráfica 12. Motivos de las relaciones de las PyMES con Otras Empresas (productoras, proveedoras)

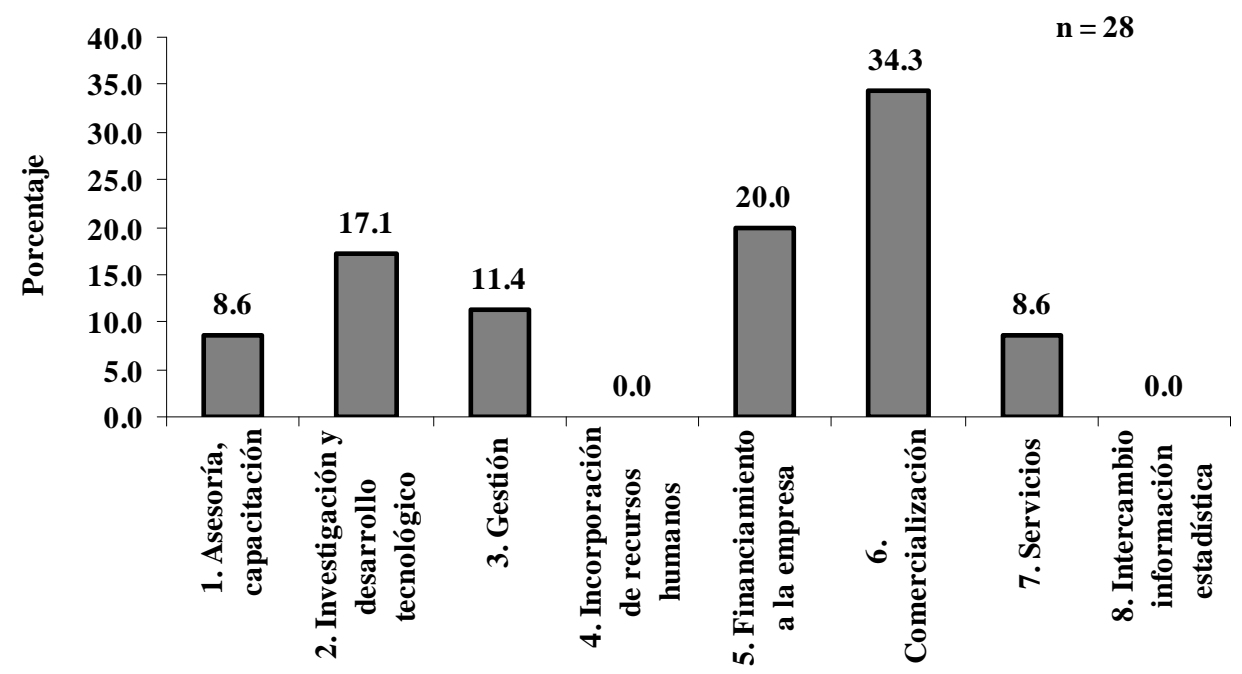

Fuente: Entrevistas a PyMES acuícolas de la región Noroeste de México.

\section{Interacciones tipo 5, academia-gobierno-empresa}

Por lo que se refiere a las interacciones tipo 5, es decir, las que involucran tanto a actores del sector académico, con del productivo y del gobierno, en los últimos años se han creado un conjunto de instituciones y organismos descentralizados, con objeto de lograr la articulación entre estos tres actores. Como ejemplo de ello, podemos mencionar la creación de los Comités de Sanidad Acuícola, que son organismos tripartitas integrados por representes del gobierno, la academia y la empresa, que tienen la función de hacer el diagnóstico patológico, monitoreo y tratamiento de los cultivos. Las unidades productivas aportan una cuota, los investigadores investigan, diagnostican las patologías y prescriben, mientras que el 
REDES- Revista hispana para el análisis de redes sociales

Vol.17,\#6, Diciembre 2009

http://revista-redes.rediris.es

gobierno media entre ellos y coordina el funcionamiento de los comités. Se trata de una articulación en red, que ha tenido un impacto positivo en la actividad acuícola de la región.

\section{Discusión}

Los resultados preliminares que presentamos en la sección anterior se centran sobre todo, en la identificación de algunas de las interacciones que se establecen entre tres diferentes actores (empresas acuícolas, universidades y centros de investigación e instituciones de gobierno).

La información recabada a partir de 31 entrevistas a PYMES acuícolas de la región noroeste y otras fuentes documentales, sugiere que en general existen un conjunto de interacciones entre los actores, con diferentes grados de intensidad, duración y frecuencia, dependiendo de los intereses, recursos y tipos de conocimiento (científico, tecnológico, administrativo, financiero, etc.) de que dispone cada uno de ellos. Así, por ejemplo, en el caso de las interacciones academia-empresa, éstas obedecieron sobre todo, a los requerimientos de recursos humanos, la ejecución de proyectos de investigación y desarrollo, la prestación de servicios, asesoría y capacitación. De igual modo, las interacciones empresa-gobierno respondieron a las necesidades de gestión y financiamiento por parte de las PYMES acuícolas. En cuanto a las interacciones empresa-empresa, las motivaciones fundamentales fueron los requerimientos de comercialización, investigación y desarrollo tecnológico y gestión de las empresas.

De nuestro análisis se desprende que tales interacciones no parecen ser muy frecuentes ni duraderas, y se han sustentado sobre todo en relaciones informales cara a cara y poca confianza mutua, teniendo un escaso impacto en desarrollos tecnológicos $u$ organizacionales de importancia en esta actividad $y$, consecuentemente, en el desarrollo regional. En otras palabras, pese a que en esta región existe un conjunto de capacidades para la generación de conocimiento en la acuacultura, tanto en universidades y centros de investigaciones como instituciones gubernamentales y empresas privadas y sociales, no se observa una sólida interacción y transferencia de conocimiento entre los actores que participan en esta actividad.

Entre los factores que - de acuerdo con nuestro primer análisis--, podrían estar limitando las oportunidades de interacción y transferencia de conocimiento entre estos distintos actores es posible mencionar: 1) la falta de confianza; 2) la ausencia redes densas entre los actores; 3) la falta de comunicación y un lenguaje común; 
REDES- Revista hispana para el análisis de redes sociales

Vol.17,\#6, Diciembre 2009

http: //revista-redes.rediris.es

4) la distancia geográfica entre las unidades productivas, las universidades, centros de investigación y las dependencias gubernamentales, y 5) la escasa capacidad de absorción de conocimientos tácitos y codificados por parte de las empresas acuícolas. Estos factores o variables, desde luego, tendrán que ser analizados en una etapa posterior de esta investigación, con otros instrumentos metodológicos.

Finalmente, aún cuando se han identificado algunas redes de conocimiento entre estos actores, éstas son débiles, la colaboración es escasa y existe poca utilización del conocimiento científico y tecnológico producido en los centros de investigación. Se trata, en suma, de una región que, no obstante contar con una serie de ventajas -tales como la proximidad geográfica, el marco institucional, el acervo de conocimiento, los recursos humanos, el sector financiero y un cierto grado de apertura, como sugeriría la teoría-, no parece haber conseguido movilizar suficientemente el capital social de conocimiento disponible localmente que contribuya a consolidar, a través de la acuacultura, un sistema regional de innovación en el noroeste de México.

\section{Conclusiones}

El trabajo que hemos presentado, forma parte de un proyecto más amplio en el cual se analiza a profundidad cómo se construyen redes de conocimiento en la acuacultura, en la región noroeste de México.

La información recabada de las entrevistas y otras fuentes documentales, sugieren que la región se caracteriza por tener una estructura de generación, transferencia y uso de conocimiento local muy desigual, con pocos flujos entre los centros de investigación y universidades hacia el sector de la acuacultura. Además, en ésta última predominan las empresas privadas que utilizan tecnología importada y conocimientos provenientes en muchos casos de empresas extranjeras.

Aunado a lo anterior, la falta de políticas y programas gubernamentales consistentes de los últimos años, centrados en las especies más comerciales (como el camarón), en detrimento de otras menos comerciales pero muy importantes para las unidades productivas sociales, han dificultado también el avance de este sector.

La actividad acuícola - como actividad económica y social- se caracteriza por una relativa complejidad, en virtud de la cantidad y calidad de los procesos involucrados para conseguir el producto final. Estos procesos van desde la producción y reproducción de semilla, los problemas de nutrición, la genética de las especies, la patología, las tecnologías que se requieren para el cultivo, los procesos de empaque y congelación, así como los impactos en el medio natural. Cabe destacar que la 
REDES- Revista hispana para el análisis de redes sociales

Vol.17,\#6, Diciembre 2009

http: //revista-redes.rediris.es

gran variedad de cultivos que se promueven en la región noroeste, plantean un espectro muy amplio de necesidades de conocimiento, ya que cada una de las especies tiene sus características propias. Con todo, no se ha detectado en la región una política que estimule una mayor interacción entre los centros de investigación y las universidades de la región, las dependencias gobierno (Federal, estatal y municipal) y los sectores productivos (privado y social). Para ello, se requiere pensar en la construcción de un sistema de conocimiento y de innovación que, mediante un cambio de actitud de los diferentes actores, favorezca la colaboración y el diseño de estrategias y políticas que fortalezcan las redes de producción, transferencia y apropiación social del conocimiento a nivel regional y/o local, así como su conexión con otras en el ámbito nacional y global.

\section{Bibliografía}

Adler, P. y Kwon S.W. (2000). "Social capital: The good, the bad, and the ugly", en Lesser, Eric (editor), Knowledge and social capital: Foundations and applications, MA, Butterworth-Heinemman, pp. 89-115.

Álvarez-Torres, P., Ramírez-Martínez, C., Orbe-Mendoza, A. (1999). Desarrollo de la Acuacultura en México y Perspectivas de la Acuacultura Rural. Red de Acuicultura Rural en Pequeña Escala. Taller ARPE, FAO-UCT, 09 al 12 Noviembre. 38 pp.

Asheim, B. T. y Lars C. (2006). "Contextualising regional innovation systems in a global learning economy: On knowledge Bases and Institucional Frameworks", en J ournal of Technology Transfer, vol. 31, pp. 163-166.

Avilés Quevedo, S. y Vázquez Hurtado, M. (2006). “Fortalezas y debilidades de la acuacultura en México", en Patricia Guzmán Amaya y Dilio Fuentes Castellanos (coordinadores), Pesca, acuacultura e investigación en México, México, Cámara de Diputados, Comisión de Pesca, CEDRSSA, pp. 69-86.

Bourdieu, P. (1979). Les trois états du capital cultural, en ARCS, No. 3, pp. 3-6.

Carta Nacional Pesquera (2004): Diario Oficial de la Federación. ConAPESCA.

Casas, R. (coord.) (2001). La formación de redes de conocimiento: una perspectiva regional desde México, Barcelona, IIS-UNAM/Anthropos.

Casas, R. (2001). “La transferencia de conocimientos en biotecnología: formación de redes a nivel local", en Casas, R. (coord.), La formación de redes de conocimiento: una perspectiva regional desde México, Barcelona, IISUNAM/Anthropos, pp. 163-240. 
REDES- Revista hispana para el análisis de redes sociales

Vol.17,\#6, Diciembre 2009

http: //revista-redes.rediris.es

Casas, R. (2003). "Enfoque para el análisis de redes y flujos de conocimiento", en Luna, M. (coord.): Itinerarios del conocimiento. Formas, Dinámicas y Contenido. Un enfoque de redes, Barcelona, IIS-UNAM / Anthropos, pp. 19-50.

Casas, R., De Gortari R. y Santos M. J. (2000). "The building of knowledge spaces in Mexico. A regional approach to networking", Research Policy, Elsevier (Amsterdam), N. 29, pp. 225-241.

Casas, R., Luna M. y Santos M. J. (2001). "Conclusiones”, en Casas, R. (coord.) (2001): La formación de redes de conocimiento: una perspectiva regional desde México, Barcelona, IIS-UNAM / Anthropos.

CIBNOR (1994). “I Reunión entre Sector productivo y Centros de Investigación dedicados al Cultivo de Camarón", Dirección General de Acuacultura de la Secretaría de Pesca, CIBNOR, Cámara Nacional de la Industria Pesquera, La Paz, B. C. S., 7-8 de octubre.

Coleman, J. (2000). "Social capital in the creation of human capital", en Partha Dasgupta y Ismael Serageldin, Social capital. A multifaceted Pespective, Washington, D. C., The World Bank, pp. 13-39.

CONACYT (1998). Historia de las Instituciones del Sistema SEP-CONACYT, Secretaría de Educación Pública / Consejo Nacional de Ciencia y Tecnología, México.

DeBresson, C. y Amesse, F. (1991). "Networks of innovators: A review and introduction to the issue", Research Policy, No. 20, pp. 262-279.

Etemad, H. y Chu H. (2004). "The dynamic impact of regional clusters on international growth and competition: some grounded propositions", en Hamid Etemad (editor), International entrepreneurship in small and medium siza enterprieses. Orientation, environment and strategy, UK, Edward Elgar, pp. 39-56.

Etzkowitz, H. (1994). “Academic-industry relations: a sociological paradigm for economic development", en Leydesdorff, Loet y Peter Van den Besselaar, Evolutionary Economics and Chaos Theory. New Directions in Technology Studies, Londres, Pinter, pp. 139-151.

Etzkowitz, H. y Uzzi, B. (1996). "Knowledge based economic and social development: the Triple Helix of regional cooperation among universities, industry and government", Universidad Estatal de Nueva York, (mimeo).

Freeman, C. (1987). Technology, policy and economic performance.Lessons from Japan, Londres, Pinter. 
REDES- Revista hispana para el análisis de redes sociales

Vol.17,\#6, Diciembre 2009

http://revista-redes.rediris.es

Fountain, J. (1998). "Social capital: A key enabler of innovations in science and technology", en Investing in Innovations: Toward A Consensus Strategy for Federal Technology Policy, Cambridge, The MIT Press, pp. 1-14.

Gibbons, M., Limoges C., Nowotny H ,. Schwartzman S., Scott P. y Trow M.(1994). The New Production of Knowledge. The dynamics of science and research in contemporary societies, Londres, SAGE.

Granoveter, M. S. (1984). "The strenght of weak ties", en American Journal of Sociology, vol.78, no.6, pp. 1360-1380.

Grootaert, Ch. y Th. van Bastelaer (ed.) (2002). Understanding and Measuring Social Capital, Washington D. C., World Bank.

Gross, J.y Stren, R. (2001). “Knowledge networks in global society: Pathways to development", en Gross, J., Estren, R.y Maclean M., Networks of knowledge, Canada, IPAC, IAPC, University of Toronto Press, pp. 3-28.

Guarneros y Pérez, R. (2006). “Análisis económico de la actividad pesquera y acuícola", en P. Guzmán Amaya y D. Fuentes Castellanos (coordinadores), Pesca, acuacultura e investigación en México, México, Cámara de Diputados, Comisión de Pesca, CEDRSSA, pp. 145-156.

Gunasekara, Ch. (2006). "The generative and developmental roles of universities in a regional innovation systems", Science and Public Policy, vol. 33, No.2, marzo, pp. 136-150.

Hakansson, H.(1987). Industrial technological development: A network approach. Londres, Croom Helm.

Hedstrom, P. y Swedberg R. (1994). "Introduction to the Special Issue on Social Network Analysis", Scandinavian Sociological Association, Acta Sociológica, (Oslo), 37, 327-328.

Knoke, D. (1990). Political Networks. The Structural Perspective, Cambridge, Nueva York, Port Chester, Melbourne Sydney, Cambridge University Press.

Lesser, E. (2000). "Leveraging social capital in organizations", en Lesser, Eric (editor), Knowledge and social capital: Foundations and applications, MA, Butterworth-Heinemman, pp. 3-16.

Lluch Cota, D. y Hernández Vázquez, S. (coords.) (2006). Desarrollo sustentable de la pesca en México. Orientaciones estratégicas, México, $\mathrm{ClB} /$ Senado de la República. 
REDES- Revista hispana para el análisis de redes sociales

Vol.17,\#6, Diciembre 2009

http://revista-redes.rediris.es

Luna, M. (coord.) (2003). Itinerarios del conocimiento. Formas, Dinámicas y Contenido. Un enfoque de redes, Barcelona, IIS-UnAM / Anthropos.

Lundvall, B. A. (1992). National Systems of Innovation: Towards a Theory of Innovation and Interactive Learning, Londres, Pinter Publishers.

Lundvall B. A., y Borras, S. (1997). The globalising learning economy: Implications for innovations policy, Luxembourg, European Communities.

Mitchell, J. C. (1973). "Networks, norms and institutions", en Boissevain, J. y Mitchell, J.C. (eds.), Network Analysis, Studies in Human Interaction, La Haya, Mouton, pp. 2-35.

Nahapiet, J.y Ghoshal, S. (2000). "Social capital, intellectual capital, and the organizational advantage", en Lesser, Eric (editor), Knowledge and social capital: Foundations and applications, MA, Butterworth-Heinemman, pp. 119-158.

Nelson, R. y Winter, S. (1982). An evolutionary theory of economic change, Cambridge, MA, The Belknap Press of Harvard University Press.

Putnam, R. (ed.) (2003). El declive del Capital Social. Un estudio internacional sobre las sociedades y el sentido comunitario, Galaxia Gutenberg, Círculo de Lectores, Bercelona ( 1 a edición en español).

Rosenberg, N. (1992). Inside the black box, Cambridge, MA, Cambridge University Press.

Rózga, R. (2002). “Entre globalización tecnológica y contexto nacional y regional de innovación", en Corona L. y R. Hernández (coord.), Innovación, Universidad e Industria en el desarrollo Regional, CIECAS-IPN / Fundación Friedrich Ebert Stiftung / UnAM, México, pp. 29-50.

Schuetze, H. G. (1996). “Innovation Systems, regional development and the role of universities in industrial innovation", en Industry \& Higher Education, abril, pp. 7178.

Schamp, E. W., y Lo V. (2003). “knowledge, leaning and regional development: A introduction, en Vivien Loy Eike W. Shamp (eds.), Knowledge, learning and regional development, Hamburgo-Londres, Lit Verlag Münster.

SEMARNAT (2005). Informe de la situación del medio ambiente en México. Compendio de estadísticas ambientales, México.

Von Hippel, E. (1988). The sources of innovation, Nueva York, Oxford University Press. 